\title{
Research Article Identifying Criteria for a Physical Literacy Screening Task: An Expert Delphi Process
}

\author{
Heather L. Rotz ${ }^{1}$ (i) , Anastasia Alpous ${ }^{1}$, Charles Boyer ${ }^{1}$, Patricia E. Longmuir ${ }^{1,2^{*}}$ (i) \\ ${ }^{1}$ Children's Hospital of Eastern Ontario Research Institute, Ottawa K1H 8L1, Canada \\ ${ }^{2}$ University of Ottawa, Faculty of Medicine and Faculty of Health Sciences, Ottawa K1H 8M5, Canada
}

\author{
Article Information \\ History: \\ Received: June 3, 2020 \\ Accepted: October 15, 2020 \\ Published: October 16, 2020 \\ Keywords: \\ Assessment \\ Development \\ Physical literacy
}

\begin{abstract}
Objectives: REACH (Recreation, Education, Allied-health, Coaching, Healthcare) leaders support children's physical literacy journey in diverse settings. This project sought physical literacy screening tool criteria that REACH leaders could use to assess children.

Methods: A 3-round expert Delphi process sought consensus (75\% of participants stating agree/strongly agree) regarding physical literacy screening. Group discussions (Round 1) identified screening issues. Qualitative analyses represented the issues as statements. Experts rated each statement (5-point Likert scale) in Rounds 2 and 3. Mean Round 2 rating for each statement was provided in Round 3.

Results: 53 experts were invited to participate with 37 ( $63 \%$ female, mean career length $=16$ years) providing consent. Each round comprised at least 7 experts with primary/secondary expertise for each sector. Round 1 identified 60 criteria and 27 potential screening tasks, which were represented in 90 statements. Consensus was achieved for 44/90 statements in Round 2 and 51/90 statements in Round 3.

Conclusions: Expert consensus suggests that physical literacy screening should utilize both objectively measured tasks and questionnaires. Encompassing multiple facets of physical literacy, including motor competence, motivation, strength, endurance, and daily behavior, is important. Research is required to identify potential tasks that meet these criteria and are suitable for each REACH sector.
\end{abstract}

\section{INTRODUCTION}

$\mathrm{I}$

ncreasing sedentary behavior and decreasing physical activity are growing concerns in children's health promotion. The Canadian 24-hour Movement Guidelines recommend children aged 5-17 years perform at least 60 minutes of moderate-to-vigorous physical activity daily[1]. However, Canadian Health Measures Survey data indicate only 36\% of Canadian children and youth meet these guidelines[2]. Physical literacy has recently come to the forefront as a concept describing factors that contribute to a healthy, active lifestyle. The Canadian consensus definition of physical lit-

*Correspondence: Pat Longmuir, Children's Hospital of Eastern Ontario Research Institute 401 Smyth Road, RI\#1-214, Ottawa, ON K1H 8 L1. Tel: 613-738-3908, Fax: 613-738-4800

E-mail address: plongmuir@cheo.on.ca eracy is "the motivation, confidence, physical competence, knowledge and understanding to value and take responsibility for engagement in physical activities for life" [3].

Physical literacy is an independent indicator of cardiovascular fitness[4] and children with higher physical literacy scores are more likely to achieve the physical activity guidelines[4]. In turn, guideline compliance is associated with enhanced physical, psychological, social, and cognitive health outcomes[5,6]. Being able to identify children with lower levels of physical literacy would enable targeted interventions to decrease their sedentary lifestyle morbidity risk. To date, multiple protocols have been developed to assess physical literacy[7], but none are suitable for use as a quick screening tool by "REACH" sector leaders. REACH refers to physical literacy supportive sectors where leaders often do not have specialist physical activity training. They include: Recreation (municipal, private, camps), Education (schoolbased), Allied health (fitness, health promotion), Coaching terms of the Creative Commons Attribution License (http://creativecommons.org/licenses/by/4.0/), which permits unrestricted use, distribution, and reproduction in any medium, provided the original work is properly cited. 
(lessons, sport), or Healthcare (doctors, nurses, therapists). REACH sector leaders are well-suited to assess and support the physical literacy of all children.

The Canadian Assessment of Physical Literacy (CAPL) assesses four domains of physical literacy, including motivation and confidence, physical competence, daily activity behavior, and knowledge and understanding. The CAPL has high validity to measure the physical literacy of children aged 8 to 12 years[8] and each protocol has published reliability data for this age[9]. However, the CAPL requires significant time and space, and some protocols are designed to be performed by individuals with specialized training. The second edition of the CAPL (www.capl-eclp.ca) reduced examiner and participant burden, but still requires more time and space than would be appropriate for a screening test[10]. The Physical Literacy Assessment for Youth (PLAY), developed by Sport for Life, is designed to assess physical literacy for children aged 7 years and older. A sub-tool, called PLAYfun, and a shorter version, called PLAYbasic, have validity evidence[11], but only good inter-rater reliability for average measures and moderate-to-good reliability for single measures[12]. However, the PLAYfun and PLAYbasic tools only assess the motor competence component of physical competence, and are thus not sufficient to be used as stand-alone screening tools for the broader concept of physical literacy. Other assessment tools, such as Passport for Life, developed by Physical Health Education (PHE) Canada, currently have no peer-reviewed evidence for reliability or validity[7].

Based on the limitations of these existing physical literacy assessments, a new approach to screening childhood physical literacy is required. The goal of this project was to establish the criteria for a physical literacy screening tool that could quickly and effectively identify children at risk of inactive lifestyles due to significant physical literacy deficits. The ideal tool would be suitable for use by leaders across all REACH sectors, within the time and space constraints of their work environments.

\section{METHODS}

A Delphi process is a communication framework based on multiple rounds of feedback from a panel of experts, in order to systematically elicit a reliable consensus of opinion on emerging or unexplored issues. The Delphi method relies on anonymity of the participants, structured information flow, and feedback between the facilitators and participants $[13,14]$. A 3-round Delphi method was used to establish the criteria for a physical literacy screening task suitable for all REACH sectors. Round 1 group discussion notes were recorded without identifying information. Responses for Rounds 2 and 3 were de-identified with coded ID numbers so that results could be tabulated without identifying specific participants. The study protocol was approved by the Research Ethics Board of the Children's Hospital of Eastern Ontario (\#13/124X), and all participants provided written informed consent.

\section{Round 1}

Participants $(n=32)$ with expertise in research, education, allied health, coaching, or healthcare, and with an interest in childhood physical activity were identified. A 1-day, in-person meeting was facilitated by PL, CB, AA with assistance from 2 graduate students. The research team shared results of an environmental scan outlining current physical literacy assessment tools, including the Canadian Assessment of Physical Literacy (CAPL). A group discussion identified goals and criteria for potential screening tasks, and which domains of physical literacy (motivation, knowledge, behavior, and physical competence) should be reflected. The panel was then divided into five sub-groups. Each sub-group had 30 minutes to identify specific tasks that should be investigated as a potential screening task for children's physical literacy. These discussions were unstructured, and each subgroup was comprised of 5 or 6 experts plus one designated note-taker. Experts were assigned to a sub-group to ensure that each group had representation from each REACH sector. The benefit of having multiple sub-groups rather than a single group discussion. It is easier for each person to contribute their ideas in a small group of people. Combining the information from each small group makes it more likely that diverse points of view are recognized and considered. Two researchers (AA, CB) utilized qualitative research methods to independently analyze the meeting notes from the presentation sessions, large group discussion, and 5 small group discussions. Comments regarding the criteria for a screening task were identified. Based on their expertise in kinesiology (i.e., graduate degree) and experience assessment physical literacy, researchers identified each comment and categorized it by the physical literacy domain represented (overall, physical competence, motivation and confidence, knowledge and understanding, daily behavior) and whether it related to the task performed (e.g., balance on one leg) or the testing environment (e.g., small clinic room). Similar comments were grouped together to represent important concepts. Three researchers (CB, AA, PEL) then met to review the identified concepts, ensure consistency between the two analyses, and identify important themes. A list of statements outlining goals and criteria for potential physical literacy screening assessment tasks was compiled based on the identified themes.

\section{Round 2}

The list of statements developed from the analyses of Round 1 responses was circulated electronically for Round 2. Delphi participants were asked to rate each statement using a 5-point Likert scale (strongly disagree, disagree, neutral, agree, and strongly agree). A priori, the researchers defined positive consensus as $\geq 75 \%$ of participants responding "agree" or "strongly agree" to a statement. It was felt that this would identify tasks and criteria that a majority of experts agreed were important. Similarly, negative consensus was defined as $\geq 75 \%$ of participants responding "disagree" 
or "strongly disagree" to a statement. Non-consensus statements were defined as those that did not meet either positive or negative consensus criteria. Frequency tabulations determined the degree of consensus for each statement and mean statement ratings were calculated. Additional questions regarding length of time for screening, space requirements for screening, and time for interpretation of results were also included.

\section{Round 3}

The group mean was calculated for each statement based on 1 (strongly disagree) to 5 (strongly agree) points being assigned for each response. Statements from Round 2 were then recirculated to participants, accompanied by the group mean \pm standard deviation and their previous individual response. Participants could then retain or change their original response based on further reflection and their knowledge of the group mean response. Final response distributions, to be used to guide future research, were calculated using the Round 3 responses. The same criteria used to define consensus in Round 2 were applied to the Round 3 responses.

\section{Data Analyses}

Qualitative inductive thematic analysis was used to identify key themes and statements from the written text summarizing the meeting discussions (presentations, large group, 5 small groups). All of the written text was reviewed multiple times by two researchers (CB, AA), and comments related to screening tasks or criteria were identified. Concepts were identified to represent similar comments. Three researchers (CB, AA, PEL) then met to review the identified concepts, ensure consistency in the two analyses, and combine concepts into important themes.
For Rounds 2 and 3, points were awarded to each response: 1=Strongly disagree, 2=Disagree, 3=Neutral, 4=Agree, $5=$ Strongly agree. Frequency distributions summarized the responses to each item and determined consensus. One-way ANOVA statistics were calculated for final Round 3 data to determine whether response distributions were significantly influenced by the experts' gender or REACH sector. Analyses were completed using IBM SPSS Version 26 (IBM Corp., released 2019, Armonk, NY), with statistical significance set at alpha $=0.01$ to account for the large number of comparisons.

\section{RESULTS}

\section{Participants}

Invitations were sent to 53 experts requesting their participation in the 3-round Delphi process, of whom 37 provided consent to participate. Twenty-eight participants (75.7\%) attended Round 1, 31 (83.8\%) completed round 2 , and $28(75.7 \%)$ completed round 3. Those who did not complete all three rounds either did not respond to follow-up, or withdrew from specific rounds due to time constraints. The final panel for each round was composed of at least 7 experts with primary or secondary expertise for each sector (recreation, education, allied health, coaching, and healthcare). Descriptive statistics of participants for each round are provided in Table 1.

\section{Round 1}

The facilitated large group discussion among the 28 participants identified 45 screening task criteria. In addition, the experts agreed that the screening task should be flexible and easy to administer, require limited equipment and space, be accessible to all children and REACH sectors, and be

Table 1. Descriptive Statistics of Expert Participants

\begin{tabular}{|c|c|c|c|c|}
\hline Characteristic & Descriptors & $\begin{array}{l}\text { Round } 1 \\
\mathrm{~N}(\%)=28\end{array}$ & $\begin{array}{l}\text { Round } 2 \\
\mathrm{~N}(\%)=31\end{array}$ & $\begin{array}{l}\text { Round } 3 \\
\mathrm{~N}(\%)=28\end{array}$ \\
\hline \multirow{2}{*}{ Sex } & Male & $10(35.7)$ & $11(35.5)$ & $11(39.3)$ \\
\hline & Female & $18(64.3)$ & $20(64.5)$ & $17(60.7)$ \\
\hline \multirow{5}{*}{ Area of expertise (REACH sector) } & Recreation & $7(25.0)$ & $5(16.1)$ & $3(10.7)$ \\
\hline & Education & $6(21.4)$ & $5(16.1)$ & $5(17.9)$ \\
\hline & Allied Health & $6(21.4)$ & $10(32.3)$ & $10(35.7)$ \\
\hline & Coaching & $6(21.4)$ & $4(12.9)$ & $4(14.3)$ \\
\hline & Healthcare & $3(10.7)$ & $7(22.6)$ & $6(21.4)$ \\
\hline \multirow{5}{*}{ 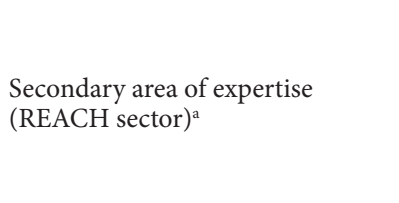 } & Recreation & - & $9(29.0)$ & $9(32.1)$ \\
\hline & Education & - & $3(9.7)$ & $3(10.7)$ \\
\hline & Allied Health & - & $1(3.2)$ & $0(0.0)$ \\
\hline & Coaching & - & $5(16.1)$ & $4(14.3)$ \\
\hline & Healthcare & - & $2(6.5)$ & $2(7.1)$ \\
\hline \multirow{3}{*}{ Career Length } & Sum (years) & 527.5 & 527.5 & 430.5 \\
\hline & Average (years) & 16.0 & 16.0 & 15.4 \\
\hline & Range (years) & $3-40$ & $3-40$ & $3-40$ \\
\hline
\end{tabular}

${ }^{a}$ Total number does not equal 31, as not all Delphi participants reported an area of secondary expertise 
Table 2. Summary of Positive Consensus for Screening Task Criteria (>75\% Agree/Strongly Agree)

\begin{tabular}{|c|c|c|c|}
\hline Statement of Screening Task to Meet & Strongly Agree / Agree & Neutral & $\begin{array}{c}\text { Disagree / } \\
\text { Strongly Disagree }\end{array}$ \\
\hline Accompanied by educational information for REACH leaders. & 27 & 1 & 0 \\
\hline Accompanied by educational information for parents. & 25 & 3 & 0 \\
\hline Suitable for the healthy population. & 25 & 2 & 1 \\
\hline Suitable for children with disabilities. & 25 & 2 & 1 \\
\hline Administered in the same way to children with/without illness or disability. & 9 & 13 & 6 \\
\hline Suitable for use with 1 child. & 18 & 9 & 1 \\
\hline Suitable for groups of $10-20$ children. & 23 & 4 & 1 \\
\hline Suitable for use with groups with more than 20 children. & 13 & 10 & 5 \\
\hline Suitable for use in all REACH sectors. & 22 & 5 & 1 \\
\hline Suitable for use in research. & 28 & 0 & 0 \\
\hline Built on existing tests/tools/questionnaires used by REACH sectors. & 17 & 10 & 1 \\
\hline Compatible with the scope of practice of each REACH sector. & 22 & 5 & 1 \\
\hline Culturally appropriate. & 25 & 2 & 1 \\
\hline Available to all children, even those in remote areas. & 26 & 2 & 0 \\
\hline An objective measurement. & 25 & 3 & 0 \\
\hline Appropriate for a child's chronological age. & $23^{\mathrm{a}}$ & 5 & 0 \\
\hline Appropriate for a child's developmental age. & $26^{\mathrm{a}}$ & 2 & 0 \\
\hline Mandated by the government. & 9 & 14 & 5 \\
\hline
\end{tabular}

${ }^{a}$ Item did not achieve consensus in Round 2

Table 3. Summary of Consensus Regarding What Facets of Physical Literacy Should be Assessed (>75\% Agree/Strongly Agree)

\begin{tabular}{|c|c|c|c|}
\hline Statement of What the Screening Task Should Assess & $\begin{array}{c}\text { Strongly Agree / } \\
\text { Agree }\end{array}$ & Neutral & $\begin{array}{c}\text { Disagree / } \\
\text { Strongly Disagree }\end{array}$ \\
\hline Motor Skill should be assessed & 27 & 1 & 0 \\
\hline Cardiorespiratory Fitness should be assessed & 26 & 2 & 0 \\
\hline Body Composition should be assessed & 5 & 13 & 10 \\
\hline Upper Body Strength should be assessed & $21^{\mathrm{a}}$ & 5 & 2 \\
\hline Lower Body Strength should be assessed & $21^{\mathrm{a}}$ & 5 & 2 \\
\hline Manual Dexterity should be assessed & 18 & 8 & 2 \\
\hline Knowledge of Physical Activity should be assessed & $21^{\mathrm{a}}$ & 7 & 0 \\
\hline Motivation towards Physical Activity should be assessed & 24 & 4 & 0 \\
\hline Core Strength should be assessed & 23 & 4 & 1 \\
\hline Flexibility should be assessed & 20 & 5 & 3 \\
\hline Daily Physical Activity Behavior should be assessed & 26 & 2 & 0 \\
\hline Daily Sedentary Behavior should be assessed & 25 & 3 & 0 \\
\hline The Physical Environment of a Child should be assessed & $22^{\mathrm{a}}$ & 5 & 1 \\
\hline The Social Environment of a Child should be assessed & $22^{\mathrm{a}}$ & 5 & 1 \\
\hline The Emotional State of a Child should be assessed & 16 & 10 & 2 \\
\hline The Motivational State of a Child should be assessed & $22^{\mathrm{a}}$ & 6 & 0 \\
\hline
\end{tabular}

a Item did not achieve consensus in Round 2

complimentary to existing resources and research. Five small groups, each with representation from multiple REACH sectors, separately developed a "top 10" list of specific screening tasks. Given the number of expert participants $(n=32)$, the variation in their expertise and experience, and the deliberate construction of small groups with representatives from each $\mathrm{REACH}$ sector, it is not surprising that there was significant heterogeneity of tasks between groups (see Supplemental File 1). However, all groups agreed that a screening assessment should consist of both physical tasks that can be objectively observed and questionnaires for self-reporting of different aspects of physical literacy. All groups also supported the inclusion of at least one task from each of the four domains within the consensus definition of physical literacy: motivation and confidence, physical competence, knowledge and understanding, and 
Table 4. Summary of Consensus Regarding the Results the Screening Should Provide (>75\% Agree/Strongly Agree)

\begin{tabular}{lccc}
\hline Statement of What Results the Screening Task Should Provide & Strongly Agree / Agree & Neutral & $\begin{array}{c}\text { Disagree / } \\
\text { Strongly Disagree }\end{array}$ \\
\hline Screening should indicate Motor Skill & 24 & 4 & 0 \\
Screening should indicate Cardiorespiratory Fitness & 23 & 5 & 0 \\
Screening should indicate Body Composition & 7 & 12 & 9 \\
Screening should indicate Upper Body Strength & 16 & 9 & 3 \\
Screening should indicate Lower Body Strength & 16 & 11 & 3 \\
Screening should indicate Manual Dexterity & 20 & 7 & 1 \\
Screening should indicate Knowledge of Physical Activity & 23 & 4 & 1 \\
Screening should indicate Motivation Towards Physical Activity & 22 & 4 & 2 \\
Screening should indicate Core Strength & 18 & 8 & 2 \\
Screening should indicate Flexibility & 25 & 3 & 0 \\
Screening should indicate Daily Physical Activity Behavior & 23 & 5 & 7 \\
Screening should indicate Daily Sedentary Behavior & 19 & 7 & 2 \\
Screening should indicate The Physical Environment of a Child & 19 & 9 & 2 \\
Screening should indicate The Social Environment of a Child & 15 & 16 & 2 \\
Screening should indicate The Emotional State of a Child & 16 & 1 \\
Screening should indicate The Motivational State of a Child & & 2 \\
\hline
\end{tabular}

${ }^{a}$ Item did not achieve consensus in Round 2

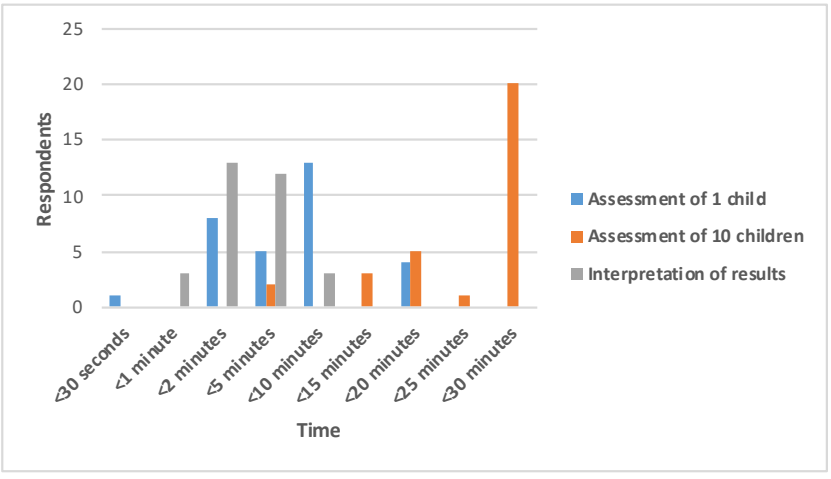

Figure 1. Recommended time required to complete screening

daily behavior. Inductive, qualitative thematic analysis produced a list of 60 criteria for an appropriate screening assessment (e.g. should be suitable for children with and without disabilities, should require minimal space, time, and resources, etc.) and a list of 27 potential screening tasks (e.g. push-ups, motivation questionnaire, vertical jump, throwing, self-reported sedentary screen time, etc.).

\section{Round 2}

Based on the list of criteria and potential tasks compiled from Round 1, a questionnaire (see Supplemental File 2) was developed to include 95 statements to be answered with a Likert scale. The statements were grouped into 6 categories: criteria for a screening tool, components of physical literacy assessed and identified, content of the screening tool, resources and training required for administration, analysis/

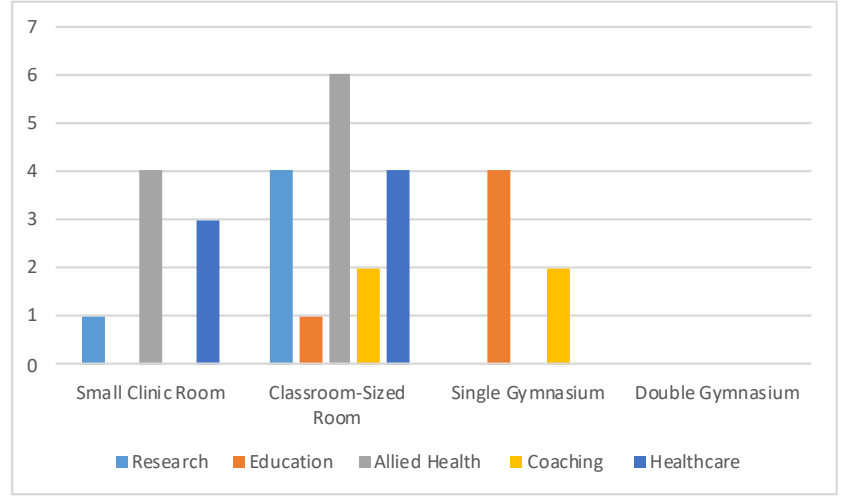

Figure 2. Recommended space required to complete screening

interpretation of results, and promotion of screening tool use. Positive consensus ( $\geq 75 \%$ of respondents) was achieved for 42 of 90 statements (46.6\%), 2 statements achieved negative consensus (2.2\%), and 48 statements (53.2\%) demonstrated non-consensus. Results for time and space requirement questions are summarized in Figures 1 and 2.

\section{Round 3}

When the questionnaire was circulated a second time with the mean scores for each question included, positive consensus ( $\geq 75 \%$ of respondents) was achieved for 47 of 90 (52.2\%) statements. Round 3 consisted of statements regarding the screening task criteria (Table 2), what aspects of physical literacy should be measured (Table 3), the results the screening task should provide (Table 4), and details of screening task test administration (Table 4). Negative 
Table 5. Summary of Consensus Regarding Specific Criteria for Screening Task Administration (>75\% Agree/Strongly Agree)

\begin{tabular}{|c|c|c|c|}
\hline Statement & Strongly Agree / Agree & Neutral & $\begin{array}{c}\text { Disagree / } \\
\text { Strongly Disagree }\end{array}$ \\
\hline Include a physical test (e.g. movement, strength, endurance). & 27 & 1 & 0 \\
\hline Include questions (e.g. motivation, activity behavior, knowledge). & 28 & 0 & 0 \\
\hline Be based on skill progressions (e.g. standing long jump to triple jump). & 13 & 12 & 3 \\
\hline Include skills that can be assessed in all REACH sectors. & 17 & 8 & 3 \\
\hline Include only one component of physical literacy (e.g. handgrip or plank). & 1 & 5 & 22 \\
\hline Be specific to one domain of physical literacy (e.g., knowledge, motivation). & 2 & 2 & 24 \\
\hline Be a circuit, reflecting all physical literacy domains. & 16 & 8 & 4 \\
\hline Maintain the domains of the Canadian Assessment for Physical Literacy. & 13 & 13 & 2 \\
\hline Be the same for each REACH sector. & 4 & 14 & 10 \\
\hline Differ by REACH sector (i.e., different leaders choose depending on need). & 18 & 8 & 2 \\
\hline Be something a child can practice on his or her own time. & 14 & 10 & 4 \\
\hline Be able to be used by parents at home. & 15 & 10 & 3 \\
\hline Be an active assessment that can be observed and scored. & 22 & 6 & 0 \\
\hline Include a questionnaire to assess motivation. & 23 & 5 & 0 \\
\hline Include a self-report measure of physical activity. & 20 & 6 & 2 \\
\hline The physical literacy screening task(s) should include only 1 item. & 0 & 3 & 25 \\
\hline The physical literacy screening task(s) should include no more than 3 items. & 2 & 10 & 16 \\
\hline The physical literacy screening task(s) should include no more than 5 items. & 13 & 11 & 4 \\
\hline Equipment needed should fit in a backpack or tool kit. & 21 & 6 & 1 \\
\hline Equipment needed should already be accessible to each REACH sector. & $26^{\mathrm{a}}$ & 1 & 1 \\
\hline Feasible using existing resources of each REACH sector. & 21 & 5 & 2 \\
\hline Leaders should have resources to encourage physical literacy screening. & 24 & 3 & 1 \\
\hline REACH leaders can implement screening without additional training. & 6 & 13 & 9 \\
\hline REACH leaders implement screening with review of written materials only. & 16 & 9 & 3 \\
\hline REACH leaders implement after webinar \& written material review. & 20 & 7 & 1 \\
\hline In-person training should be provided before implementing screening. & 3 & 13 & 12 \\
\hline Physical literacy screening task(s) should only require one staff member. & 17 & 8 & 3 \\
\hline Staff/volunteers should receive information about screening importance. & 26 & 2 & 0 \\
\hline Physical literacy screening provide results useful to REACH leaders. & 27 & 1 & 0 \\
\hline Screening results should follow children across all ages (>1 assessment). & 25 & 3 & 0 \\
\hline Screening timing should match existing contacts for each REACH sector. & 22 & 5 & 1 \\
\hline A decision tree for follow-up action should be included with the screening. & 26 & 1 & 1 \\
\hline Results should be accompanied by information for leaders and parents. & 26 & 0 & 2 \\
\hline Screening results should be compatible with the electronic medical record. & 12 & 15 & 1 \\
\hline Screening results should be in a database available to REACH leaders. & 20 & 6 & 2 \\
\hline Resources need to be available for follow-up after screening results. & 26 & 1 & 1 \\
\hline Allied-health professional referrals should be available after screening. & 24 & 2 & 2 \\
\hline A publication/promotion plan should be developed with the screening. & 26 & 2 & 0 \\
\hline Screening should be publicized so REACH sectors know the benefits. & 27 & 0 & 1 \\
\hline Screening task(s) do not require promotion, just word-of-mouth awareness. & 3 & 1 & 24 \\
\hline
\end{tabular}

a Item did not achieve consensus in Round 2

consensus was achieved for 4 statements (4.3\%). The four statements that achieved negative consensus (i.e., consensus of disagreement) indicated that the screening task would require promotion/publication and should include more than one task, more than one component of physical literacy, and not be specific to one domain of physical literacy. 39 statements (43.5\%) had diverse responses and did not demonstrate consensus. These diverse opinions were also reflected in the feedback regarding the time and space required to complete the screening task(s). The most frequent response $(13 / 28,46 \%)$ regarding the time required to screen one child was less than 10 minutes, but 11 (39\%) experts thought that screening should be completed in less than 2 minutes (Figure 1). Most respondents felt that it would be appropriate to screen a group of 10 children in $25-30$ minutes $(n=20,71 \%)$. Interpretation of results 
was recommended to be less than 2 minutes $(n=14,50 \%)$ or 5 to 10 minutes $(n=14,50 \%)$. The size of space required (Figure 2) was recommended as a small clinic room $(n=9$, $32 \%)$, a classroom $(n=14,50 \%)$ or a gymnasium $(n=5,18 \%)$. Participant gender did not significantly influence the results. Participant REACH sector also did not influence most results, with two exceptions. Leaders from the education and allied-health were less likely to agree $(p=0.003)$ the screening task should be appropriate for the child's chronological age. Whether the screening task should measure body composition also differed by sector, with leaders from alliedhealth and coaching being less likely $(\mathrm{p}=0.01)$ to agree that the measurement of body composition was important (Table $5)$.

\section{DISCUSSION}

Leaders from the Recreation, Education, Allied health, Coaching and Healthcare (REACH) sectors are strategically situated to proactively identify and support children at risk of low engagement in physical activity. To target supportive resources to children with the greatest need, REACH leaders require a physical literacy screening tool that can be used effectively in these settings. Through a Delphi process, this work identified the criteria for a physical literacy screening tool based on expert consensus. The ideal screening tool should assess multiple domains of physical literacy, including physical competence, knowledge and understanding, and motivation. It should be accompanied by educational information, suitable for use in all REACH sectors, based on objective measurements, appropriate for the chronological and developmental age of the participants and for use with children with and without disabilities. There was consensus that the screening tool should provide an indication of the child's motor skill, cardiorespiratory fitness, core strength, activity motivation and their active and sedentary daily behavior. To achieve these goals, the screening tool should be comprised of both active movement assessments and questionnaires assessing motivation and/or knowledge. The screening results should also provide information useful to REACH leaders and parents, and provide a decision tree and referral resources for follow-up action when needed.

While a majority of the respondents $(20 / 28,71 \%)$ felt that 25 to 30 minutes would be a suitable time for screening a group of 10 children, the Delphi panel was unable to achieve consensus regarding the amount of time to screen one child or the space that should be required. The respondents were fairly evenly split between recommending that screening one child be completed in less than 2 minutes or that requiring up to 10 minutes would be appropriate. Similarly, many participants felt that the assessment tool should be suitable for administration in a small clinic space, while others indicated that a classroom-sized space would be appropriate. Surprisingly, the differences among participants were largely unrelated to their gender, REACH sector or years of service. Males tended to allow a longer time to screen one child $(\mathrm{r}=0.35, \mathrm{p}=0.07)$ and the time required to assess a group of 10 children was higher among leaders with fewer years of experience $(\mathrm{r}=0.37, \mathrm{p}=0.05)$. REACH sector was not associated with recommendations regarding the time or space required for screening $(\mathrm{r}<0.15, \mathrm{p}>0.50)$ suggesting that the same screening tool could be suitable across sectors and settings.

That many experts recommended the screening be completed in less than 2 minutes would seem to somewhat contradict their recommendations for screening task content. Content recommendations focused on objective measurements and emphasized that the screening should incorporate more than one task, and assess more than one physical literacy domain. There was consensus that the screening task should provide an indication of motor skill, cardiorespiratory fitness, physical activity motivation, core strength and daily active and sedentary behaviors. For each of these 6 items to be objectively measured in 2 minutes would require each task to be a maximum of 20 seconds or the use of more complex tasks that combine multiple measures (e.g., a circuit of stations). Daily active and sedentary behaviors require multiple days of monitoring to obtain accurate and objective measures[15]. Similarly, measures of cardiorespiratory fitness require at least 2 to 3 minutes of steady state exercise[16]. Daily activity, screen time and cardiorespiratory fitness could be assessed quickly by asking children to self-report, however it is recognized that self-report data represent perceived activities rather than actual participation. Investigations of potential screening tasks will need to give careful consideration to balancing the desire for a more comprehensive, objective screening tool with the desired timeline.

The screening task content aligns closely with previously recommended components of physical literacy. The Canadian consensus definition of physical literacy[17], which aligns with the physical literacy philosophical writings of Whitehead[18], emphasizes the importance of physical competence (motor skill, strength, endurance, etc.), knowledge and motivation as it relates to the achievement of an active lifestyle (daily behavior). A previous Delphi process to establish the components of a detailed assessment of physical literacy[8] also emphasized the importance of movement skill, strength and cardiorespiratory fitness. The second edition of the Canadian Assessment of Physical Literacy also increased the importance of physical activity motivation within the overall physical literacy score[10]. The Canadian Agility and Movement Skill Assessment is an agility course that assesses fundamental, complex and combined movement skills with the median completion time for each trial of 17 seconds. Two practice trials and two timed/scored trials are required, for a total time requirement of 1.5 minutes per child[19]. The PLAYbasic assessment focuses on five motor tasks, running, jumping, throwing, kicking, and balance[20], which also align with the motor assessment consensus criteria developed by this Delphi panel. Both of these items could potentially be used as part of an effective screening task, although the moderate-to-good test-retest reliability reported for the PLAY tools[12] would 
be of concern. The motivation assessment of the Canadian Assessment of Physical Literacy-2[20] or the Children's Self-perceived Adequacy and Predilection for Physical Activity[21] are existing measures of children's motivation for physical activity.

\section{Strengths and Limitations}

While, an effort was made to have equal representation from each REACH sector on the panel, agreement for participation and completion of all rounds of the Delphi protocol could not be controlled. This resulted in unequal distribution of experts from the different sectors (Tables 1 \& 2) which may have introduced bias into the recommendations and feedback. Variability in the requirements for administration of a screening test may be due to different resources available among $\mathrm{REACH}$ sectors, although our analyses did not indicate a systematic difference in recommendations by sector. At the start of the Round 1 meeting, the researchers presented the Canadian consensus definition of physical literacy, which matches that of the International Physical Literacy Association, as the framework for the discussions. It was recognized that there are multiple definitions for the concept of physical literacy, and therefore the Canadian consensus definition was presented and discussed in order to provide a common knowledge base among the participating experts. However, use of this definition may have influenced the direction of the recommendations provided. Finally, the Round 3 panel included more females $(n=17,61 \%)$, with higher representation from allied health $(n=10,36 \%)$ and healthcare $(n=6,21 \%)$ than other sectors $(n<5)$. In addition, over half of the experts were from the allied health or healthcare sector, which may explain why there was a focus on cardiorespiratory fitness and body strength, since these are well-known indicators of physical health.

\section{CONCLUSIONS}

A physical literacy screening tool would enable leaders in recreation, education, allied health, coaching, and healthcare to identify children with limited capacity for a healthy, active lifestyle (i.e., low physical literacy). Expert consensus suggests that such an assessment should encompass multiple facets of physical literacy, such as motor competence, motivation, strength, endurance, and daily behavior. Objectively observed physical tasks could be combined with subjective questionnaire responses, as appropriate for each factor being assessed. Additional research is required to evaluate the efficacy of potential tasks meeting these criteria, which can be completed quickly and are suitable for use in each of the REACH sectors. The reliability of screening tasks results, their validity relative to an in-depth physical literacy assessment[10], and the generalizability for screening different ages and children with/without medical conditions or disabilities should also be evaluated.

\section{ACKNOWLEDGEMENTS}

This study was undertaken at the University of Ottawa and The Children's Hospital of Eastern Ontario. The authors thank the voluntary expert participants in the Delphi panel for their contribution to this research. This project was made possible through the financial support of the Government of Ontario.

\section{Authors' Contributions}

HLR contributed interpretation of results and manuscript writing. AA contributed to data collection. CB contributed to data collection. PEL designed the study; contributed to data collection; completed the data analysis; contributed to interpretation of results. All authors have read and approved the final version of the manuscript.

\section{Conflicts of Interest}

The authors declare no conflict of interest.

\section{REFERENCES}

1. Canadian Society for Exercise Physiolgoy. Canadian 24-hour Movement Guidelines for Children and Youth: An integration of physical activity, sedentary behaviour, and sleep. 2017; https://csepguidelines.ca/wp-content/ themes/csep2017/pdf/Canadian24HourMovementGuidelines2016_2.pdf (Accessed Oct 7, 2020)

2. Roberts KC, Xiaoquan Y, Carson V, et al. Meeting the Canadian 24-hour movement guidelines for children and youth. Health Rep. 2017; 28(10):3-7.

3. Tremblay MS, Costas-Bradstreet C, Barnes JD, et al. Canada's physical literacy concensus statement: process and outcome. BMC Public Health. 2018; 18:1034.

4. Lang JJ, Chaput JP, Longmuir PE, et al. Cardiorespiratory fitness is associated with physical literacy in a large sample of Canadian children aged 8 to 12 years. BMC Public Health. 2018; 18:1041.

5. Carson V, Lee EY, Hewitt L, et al. Systematic review of the relationships between physical activity and health indicators in the early years (0-4 years). BMC Public Health. 2017; 17:854.

6. Carson V, Chaput JP, Janssen I, Tremblay MS. Health associations with meeting new 24-hour movement guidelines for Canadian children and youth. Prev Med. 2017; 95(1):7-13.

7. Robinson DB, Randall LR. Marking Physical Literacy or Missing the Mark on Physical Literacy? A Conceptual Critique of Canada's Physical Literacy Assessment Instruments. Meas Phys Educ Exerc Sci. 2017; 21(1):4055.

8. Francis CE, Longmuir PE, Boyer C, et al. The Canadian Assessment of Physical Literacy: Development of a Model of Children's Capacity for a Healthy, Active 
Lifestyle Through a Delphi Process. J Phys Act Health. 2016: 13(2):214-22.

9. Longmuir PE, Boyer C, Lloyd M, et al. The Canadian Assessment of Physical Literacy: methods for children in grades 4 to 6 (8 to 12 years). BMC Public Health. 2015; 15(1):767.

10. Longmuir PE, Gunnell KE, Barnes JD, et al. Canadian Assessment of Physical Literacy Second Edition: A streamlined assessment of the capacity for physical activity among children 8 to 12 years of age. BMC Public Health. 2018; 18 (Suppl 2):1047.

11. Cairney J, Veldhuizen S, Graham JD, et al. A Construct Validation Study of PLAYfun. Med Sci Sports Exerc. 2018; 50(40):855-862.

12. Stearns JA, Wohlers B, Mchugh TF, Kuzik N, Spence JC. Reliability and Validity of the PLAY fun Tool with Children and Youth in Northern Canada. Meas Phys Educ Exerc Sci. 2019; 23(1):47-57.

13. Fletcher AJ, Marchildon GP. Using the delphi method for qualitative, participatory action research in health leadership. Int J Qual Methods. 2014; 13(1):1-18.

14. Okoli C, Pawlowski SD. The Delphi method as a research tool: An example, design considerations and applications. Inf Manag. 2004; 42(1):15-29.

15. Tudor-Locke C, Burkett L, Reis JP, Ainsworth BE, Mac- era CA, Wilson DK. How many days of pedometer monitoring predict weekly physical activity in adults? Prev Med. 2005; 40 (3):293-8.

16. Bar-Or O, Rowland TW. Pediatric Exercise Medicine: From Physiologic Principles to Healthcare Application. Vol. 2nd. Champaign, IL. Human Kinetics. 2004, p 1-500.

17. International Physical Literacy Association. The Definition of Physical Literacy. 2014; https://physicalliteracy. $\mathrm{ca} /$ physical-literacy/. (Accessed Oct 8, 2020)

18. Longmuir PE, Boyer C, Lloyd M, et al. Canadian Agility and Movement Skill Assessment (CAMSA): Validity, objectivity, and reliability evidence for children 8-12 years of age. J Sport Heal Sci. 2017; 6(2):231-40.

19. Sport for Life Society. PLAY Tools: Physical Literacy Assessment for Youth. 2016. https://physicalliteracy.ca/ wp-content/uploads/2017/01/PLAYbasic-workbook. pdf. (Accessed Oct 8, 2020)

20. Gunnell KE, Longmuir PE, Woodruff SJ, Barnes JD, Belanger K, Tremblay MS. Revising the Motivation and Confidence Domain of the Canadian Assessment of Physical Literacy. BMC Public Health. 2018; 18 (Supp2).

21. Hay JA. Adequacy in and Predilection for Physical Activity in Children. Clin J Sport Med. 1992; 2 (3):192201. 